International Journal of Advances in Applied Sciences (IJAAS)

Vol. 8, No. 4, December 2019, pp. 290 292

ISSN: 2252-8814, DOI: 10.11591/ijaas.v8.i4.pp290-292

\title{
Optimization of transmission signal by artificial intelligent
}

\author{
Hassan Farhan Rashag, Mohammed H. Ali
}

Technical Institute of Babylon, Al-Furat Al-Awsat Technical University, Iraq

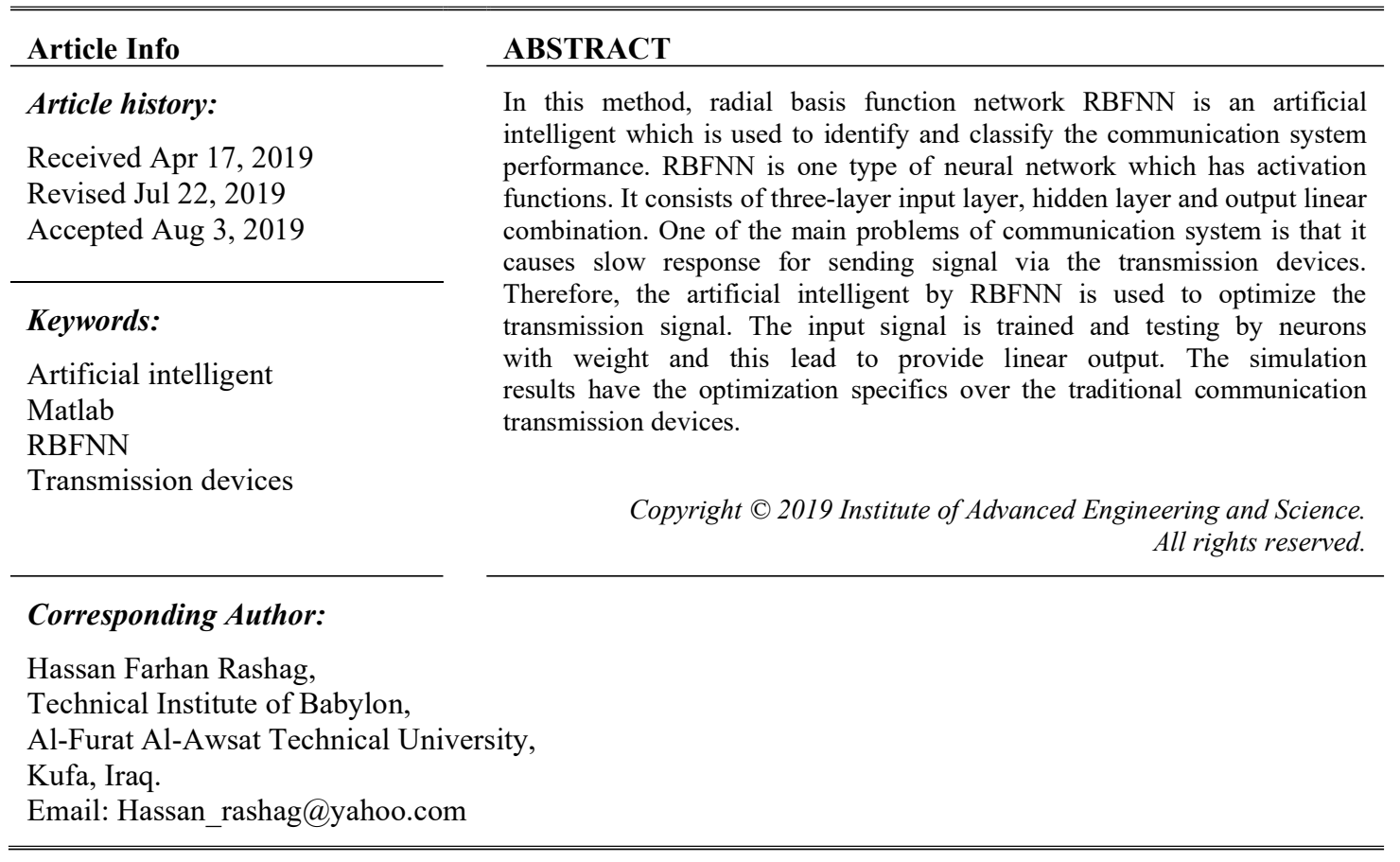

\section{INTRODUCTION}

The concept of communication system is that sending the message at one point either exactly or approximately to another point [1]. In addition, the reliably of transmitting the message from the transmission source to a destination system based on channel by transmitter and receiver devices is discussed [2]. In order to derive the theoretically optimal solution in practice, source and receiver were consequently separated into numerous treating systems. This application is called sub-optimal [3, 4]. The sub-optimal has the benefit that any element can be separately evaluated and improved which gets actual effective and constant systems which is existing nowadays. Many reports focused on the optimization for transmit the signal with more effective [5-7]. Though recently, structures are improved over the previous years and it appears hard to achieve good system but any kind of station no need for calculated exhibiting with examination. [8, 9]. Other researchers developed method to enhance the signals features by using multiple cascades for transmission devices but this method has drawbacks like slow response and distorted carrier waves [10-12].

\section{SIMULATION RESULTS}

In this method, the RBFNN is chosen to optimize the transmission signals because it has many features over the neural network. These features are classification and identification the input signal by input and hidden layer to provide linear output signal. The structure of RBFNN is shown in Figure 1. In addition, the receiver constellation with equalizer learning curve based on bit error rate BER is shown in Figure 2. From this figure, the system is almost enhanced at $\mathrm{BER}=0.49$. 


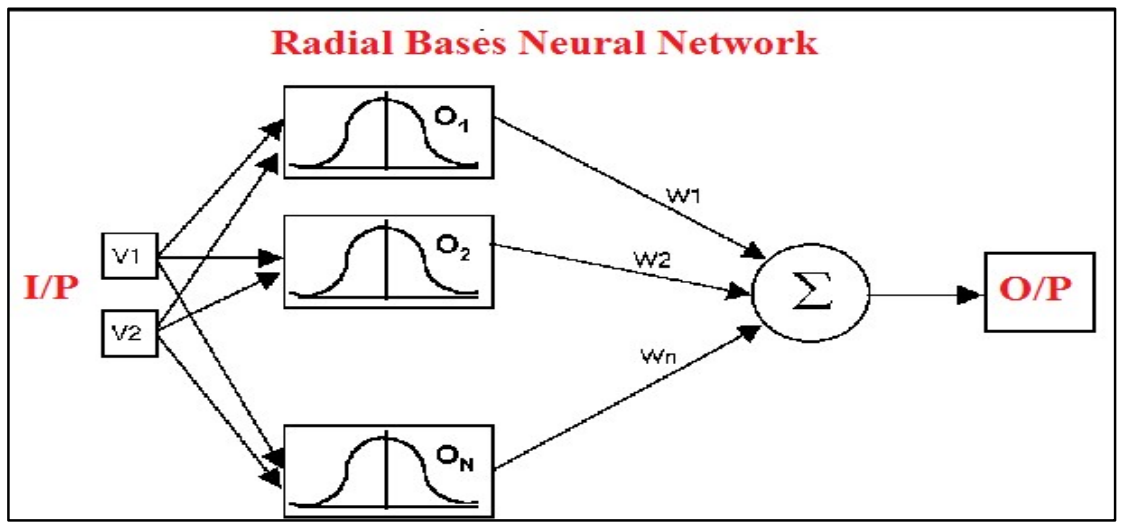

Figure 1. Structure of RBFNN

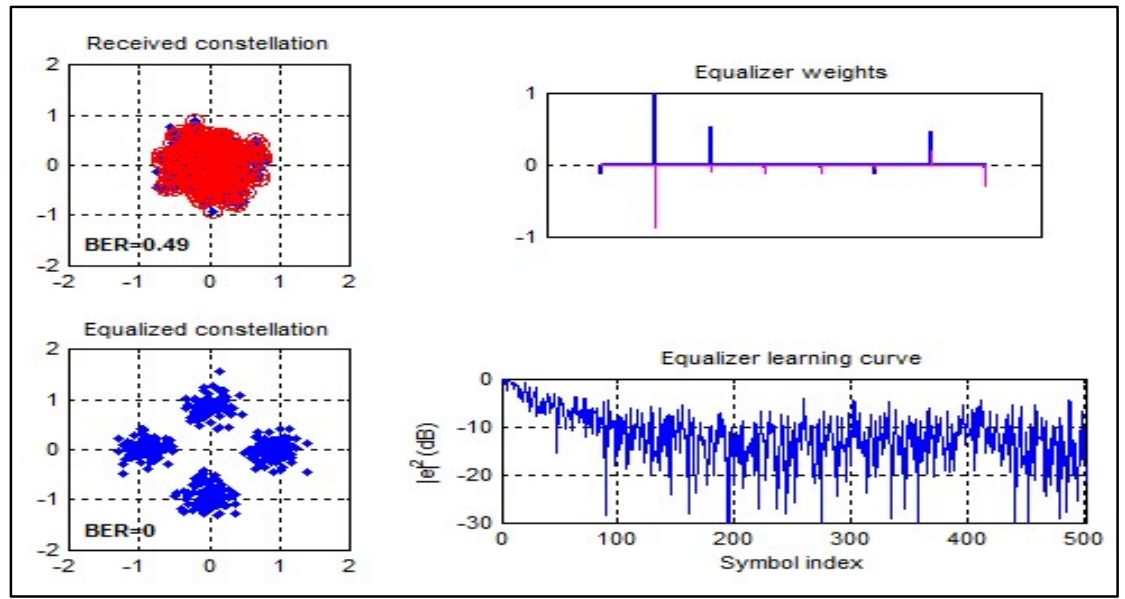

Figure 2. The receiver constellation at $\mathrm{BER}=0.49$

Figure 3 and Figure 4 show that the system with BER $=0.5,0.52$ respectively are more accurate and better for equalizer learning curve with high performance of receiver constellation.

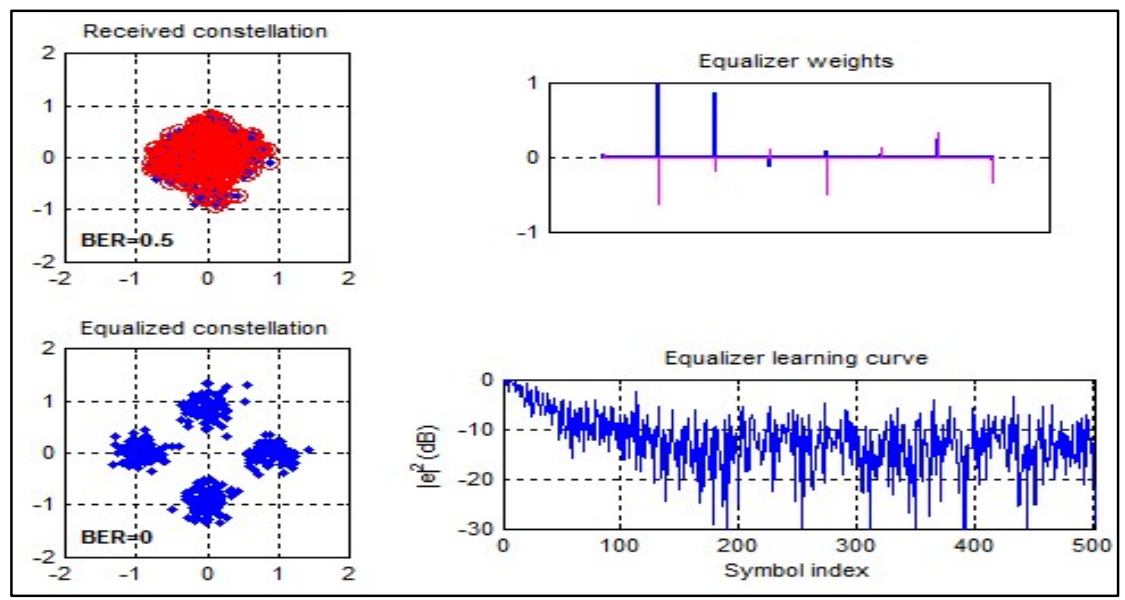

Figure 3. Receiver constellation at $\mathrm{BER}=0.5$ 


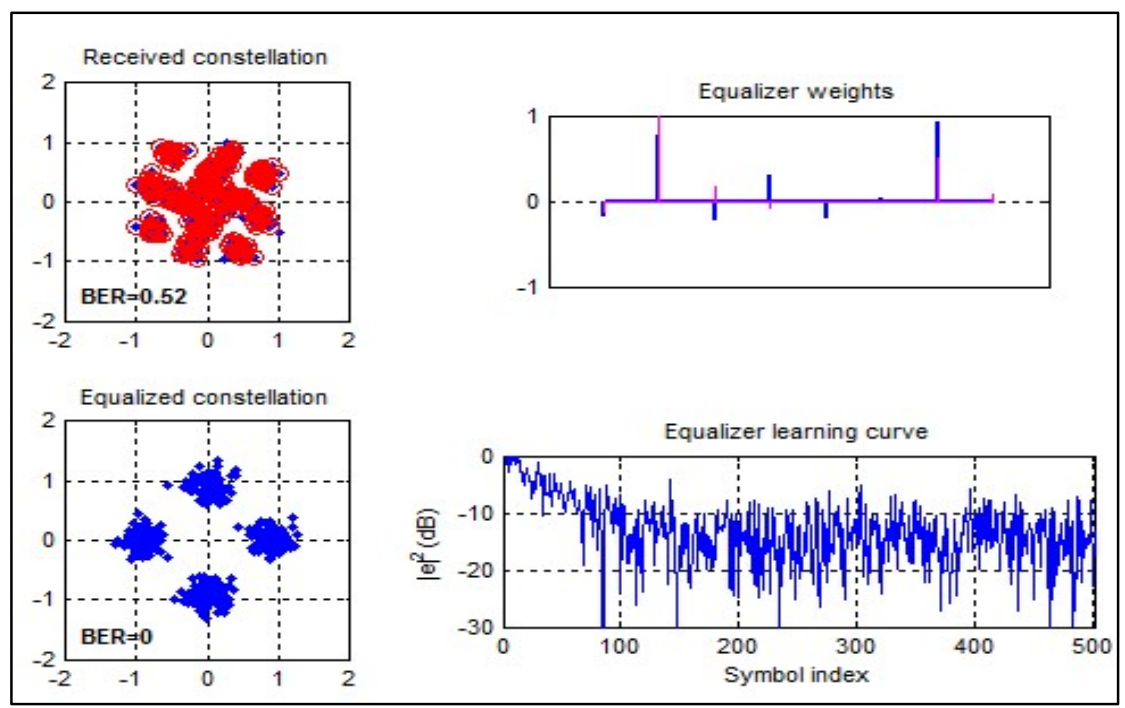

Figure 4. Equalizer learning curve at $\mathrm{BER}=0.52$

\section{CONCLUSION}

In this optimised method, the RBFNN is playing as an important tool for improved the communication system especially for transmission signal. The input signal is developed by activation function to minimize the distorted signal and to increase the response of transmission devices based on neurons and weight of hidden layer for RBFNN. The Simulink is built by tool box of matlab and the results is depended on difference values BER. finally, the system is better and optimized by high value of BER.

\section{REFERENCES}

[1] C. E. Shannon, "A mathematical theory of communication," Bell System Techical Journal, vol. 27, pp. 379-423, 623-656, 1948.

[2] E. Zehavi, "8-PSK trellis codes for a Rayleigh channel," IEEE Trans. Commun., vol. 40, no. 5, pp. 873-884, 1992.

[3] T. J. O'Shea, K. Karra, and T. C. Clancy, "Learning to communicate: Channel auto-encoders, domain specific regularizers, and attention," IEEE Int. Symp. Signal Process. Inform. Tech. (ISSPIT), pp. 223-228, 2016.

[4] T. J. O'Shea and J. Hoydis, "An introduction to machine learning communications systems," arXiv preprint arXiv:1702.00832, 2017.

[5] D. Wang, A. Khosla, R. Gargeya, H. Irshad, and A. H. Beck, "Deep learning for identifying metastatic breast cancer," arXiv preprint arXiv:1606.05718, 2016.

[6] D. George and E. A. Huerta, "Deep neural networks to enable real-time multimessenger astrophysics," arXiv preprint arXiv:1701.00008, 2016.

[7] D. Silver, A. Huang, C. J. Maddison, A. Guez, L. Sifre, G. Van Den Driessche, J. Schrittwieser, I. Antonoglou, V. Panneershelvam, M. Lanctot, et al., "Mastering the game of go with deep neural networks and tree search," Nature, vol. 529, no. 7587, pp. 484-489, 2016.

[8] M. Ibnkahla, "Applications of neural networks to digital communications: A survey," Signal Process., vol. 80, no. 7, pp. 1185-1215, 2000.

[9] M. Bkassiny, Y. Li, and S. K. Jayaweera, "A survey on machine-learning techniques in cognitive radios," Commun. Surveys Tuts., vol. 15, no. 3, pp. 1136-1159, 2013.

[10] M. Zorzi, A. Zanella, A. Testolin, M. D. F. De Grazia, and M. Zorzi, "Cognition-based networks: A new perspective on network optimization using learning and distributed intelligence," IEEE Access, vol. 3, pp. 1512-1530, 2015.

[11] R. Al-Rfou, G. Alain, A. Almahairi, et al., "Theano: A python framework for fast computation of mathematical expressions," arXiv preprint arXiv:1605.02688, 2016.

[12] M. Abadi, et al., "TensorFlow: Large-scale machine learning on heterogeneous distributed systems," arXiv preprint arXiv:1603.04467, 2016. [Online]. Available: http://tensorflow.org 